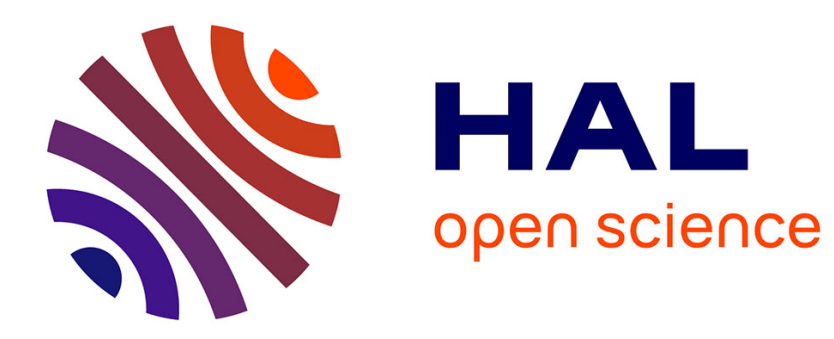

\title{
L'art formulaire d'Homère dans les Histoires Vraies de Lucien
}

Pascale Brillet-Dubois

\section{To cite this version:}

Pascale Brillet-Dubois. L'art formulaire d'Homère dans les Histoires Vraies de Lucien. Gaïa - Revue interdisciplinaire sur la Grèce archaïque, 2006, 10, pp.219-234. 10.3406/gaia.2006.1500 . halshs01545600

\section{HAL Id: halshs-01545600 \\ https://shs.hal.science/halshs-01545600}

Submitted on 23 Jun 2017

HAL is a multi-disciplinary open access archive for the deposit and dissemination of scientific research documents, whether they are published or not. The documents may come from teaching and research institutions in France or abroad, or from public or private research centers.
L'archive ouverte pluridisciplinaire HAL, est destinée au dépôt et à la diffusion de documents scientifiques de niveau recherche, publiés ou non, émanant des établissements d'enseignement et de recherche français ou étrangers, des laboratoires publics ou privés. 


\section{L'art formulaire d'Homère dans les Histoires Vraies de Lucien}

\section{Pascale Brillet-Dubois}

\section{Abstract \\ Homer's formulaic artistry according to Lucian}

When imitating Homer's Odyssey in his True Stories, Lucian not only demonstrates how well he knows the text of the Homeric poems, but also how aware he is of the poet's compositional technique, which we would nowadays label as "formulaic artistry". He structures his own narration by using renewed typical scenes and creating between them, in a very Homeric fashion, a play of echoes and variations.

\section{Résumé}

L'étude de la manière dont Lucien imite VOdyssée d'Homère dans ses Histoires Vraies révèle non seulement une connaissance très précise du texte homérique, mais aussi une conscience élevée des mécanismes de composition qui sont à l'œuvre, et que les modernes désignent sous le nom d' "art formulaire". Lucien en vient à utiliser lui-même cette technique en inventant un réseau de scènes-types qui lui sont propres, dans lequel il établit, à la manière d'un aède, un jeu de reprises et de variations.

\section{Citer ce document / Cite this document :}

Brillet-Dubois Pascale. L'art formulaire d'Homère dans les Histoires Vraies de Lucien. In: Gaia : revue interdisciplinaire sur la Grèce Archaïque, numéro 10, 2006. pp. 219-234;

doi : 10.3406/gaia.2006.1500

http://www.persee.fr/doc/gaia_1287-3349_2006_num_10_1_1500

Document généré le 08/02/2017 


\title{
L'art formulaire d'Homère dans les Histoires Vraies de Lucien
}

\author{
Pascale Brillet-Dubois
}

Université Lumière - Lyon 2

Lucien, que le voyage extraordinaire raconté dans ses Histoires Vraies conduit sur l'île des Bienheureux, se fait un malin plaisir d'y soumettre à Homère quelques-uns des problèmes que celui-ci pose depuis des siècles à ses lecteurs - quelques-uns des zètèmata chers aux Alexandrins : où estil né? A-t-il écrit les vers athétisés par ses critiques? Pourquoi diable a-til commencé l'Iliade par le mot «colère»? A-t-il véritablement écrit l'Iliade avant l'Odyssée ? ' Quant à nous, s'il nous était donné, à notre tour, de gagner cette île et d'y rencontrer Lucicn, nous lui demanderions volontiers s'il se sent $\ll$ parryiste ${ }^{2} \gg$.

En matière d'études homériques, en effet, les racines alexandrines de l'école des Analystes et de l'hypercritique en général sont bien connues, mais on se demande plus rarement si la formularité de la poésie épique, mise en évidence par Milman Parry et Albert Lord, n'était pas déjà perçue et analysée dans l'Antiquité, à défaut d'être théorisée comme elle l'a été au $\mathrm{XX}^{\mathrm{e}}$ siècle. Nous tenterons ici de déterminer si la connaissance d'Homère dont Lucien fait preuve dans les Histoires Vraies s'accompagne

1. Histoires Vraies II, 20. Sur la critique sous-jacente des querelles alexandrines, voir M. Briand, «Lucien et Homère dans les Histoires Vraies: Pratique et théorie de la fiction au temps de la seconde sophistique $\gg$, Lalies, 25, 2005, p. 127-140.

2. L'idée de cette étude $m$ 'a été inspirée par les discussions avec Marie Formarier (ENS-LSH), dont j'ai dirigé les recherches de maîtrise en 2003-2004 sur le thème de la mimésis littéraire dans les Histoires Vraies, ct par les questions de Jean-Louis Léonhardt (MOM), qui m'a conviée à parler de M. Parry à son séminaire d'épistémologie, «Sciences liées au temps des hommes». Qu'ils soient ici remerciés. 
d'une sensibilité à ce que nous appelons aujourd'hui la diction formulaire et plus particulièrement au fonctionnement des scènes que nous qualifions de typiques ${ }^{3}$.

Les Histoires Vraies sont un récit de voyage fantastique à la première personne, parodiant principalement les récits historiques et ethnographiques: Ctésias, Jamboulos, Hérodote, sont les cibles explicites de Lucien, qui laisse l'identification de ses autres victimes à l'imagination du lecteur. Il les considère comme de grands menteurs, mais leur donne pour «chef de file et maître en fariboles ${ }^{4} »(\mathrm{I}, 3)$ l'Ulysse des récits aux Phéaciens. L'Odyssée est donc l'hypotexte fondamental du récit romanesque des Histoires Vraies et va lui donner un modèle de structure, bien que se superposent à lui d'autres références dont on perçoit l'influence surtout à l'intérieur des différents épisodes, dans les descriptions de batailles ou les exposés ethnographiques concernant les peuples rencontrés par Lucien. Après une déclaration de principe parodiant celles qui introduisent les ouvrages d'histoire depuis Hécatée ${ }^{5}$, dans laquelle il affirme que rien de ce qu'il s'apprête à dire n'est vrai, Lucien se lance dans le récit d'un voyage qui le mène de l'Océan vers la Lune, puis de retour sur la mer, dans le ventre d'une baleine, sur l'île des Bienheureux ou dans l'antre de Calypso. L'imitation, toujours sensible, de la narration faite par Ulysse aux chants VIII à XII de l'Odyssée justifie que l'on s'interroge sur la manière dont Lucien traite l'art formulaire homérique, tant à l'échelle du vers qu'à celle du récit.

\section{Le pastiche des vers homériques}

On trouve dans les Histoires Vraies deux prétendues citations homériques ${ }^{6}$. La première se trouve en II, 24 . Lucien, en escale dans l'île des Bienheureux, assiste à la révolte des damnés des Enfers. Les héros, alignés contre eux par Rhadamante, leur livrent un combat où s'illustrent

3. Voir par ex. W. Arend, Die typische Szenen bei Homer, Berlin, 1933; A. Lord, The Singer of Tales, Cambridge Mass., 1960 ; J.M. Foley, Homer's Traditional Art, University Park, PA, 1999; F. Létoublon, «Le récit homérique, de la formule à l'image», Europe, 865, mai 2001, p. 20-47.

4. Nous utilisons, en modifiant parfois l'usage contestable qu'elle fait des temps, la traduction de J. Bompaire (J. Bompaire (éd., trad.), Lucien: Euvres, t. 2, CUF, BellesLettres, Paris, 1998, p. 56-134).

5. Voir F. Hartog, M. Casevitz, L'bistoire d'Homère à Augustin: Préfaces des historiens et textes sur l'bistoire, Paris, Seuil, 1999.

6. Voir O. Bouquiaux-Simon, Les lectures bomériques de Lucien, Bruxelles, 1968; M. Formarier, Mimésis littéraire dans les Histoires Vraies: l'odyssée de Lucien, mémoire de maîtrise soutenu en juin 2004 à l'Université Lyon 2 sous la direction de P. BrilletDubois, p. 24 sq. 
aussi bien Achille que Socrate. Homère écrit ${ }^{7}$ sur le sujet un poème dont il confie le texte à Lucien pour qu'il le rapporte aux vivants. Malheureusement, lors de la suite des aventures de Lucien, ce texte, comme tant d'autres œuvres attribuées au Poète par la postérité, se perd et disparaît; il n'en subsiste dans la mémoire du narrateur que le premier vers:

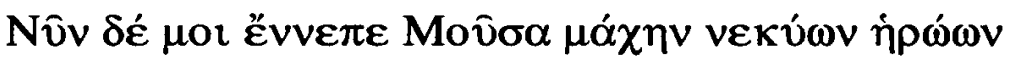

Et maintenant, ô Muse, dis-moi le combat des héros morts...

Ce vers, inventé par Lucien, est parfaitement conforme au système

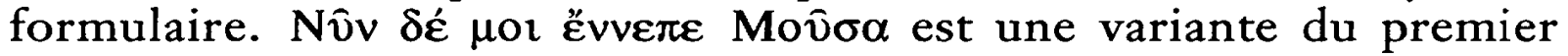
hćmistiche de l'Odyssée, et l'élément qui varie, vôv $\delta \varepsilon$, se trouve en tête de vers, comme ici, dans un grand nombre de vers épiques. Le mot $\mu \alpha ́ \chi \eta v$ est à la place que privilégie la tradition, entre les coupes trochaïque et hephthémimère ${ }^{8}$, mais au lieu d'être suivi comme c'est généralement le cas par un complément de lieu, il reçoit un complément au génitif. Celui-ci n'a pas d'équivalent dans les vers qui nous sont parve-

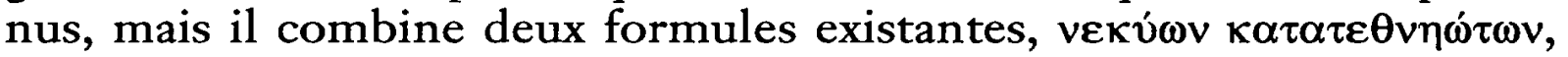
dont il adopte la place habituelle en fin de vers, et $\dot{\alpha} v \delta \rho \hat{\omega} v \dot{\eta} \rho \omega \dot{\omega} \omega v^{9}$,

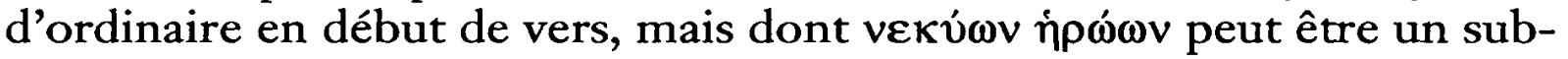
stitut métrique à une autre place. En d'autres termes, si ce plaisant pastiche ne suffit pas à prouver que Lucien analyse l'art formulaire d'Homère, il dénote néanmoins une grande familiarité avec l'épopée et une mémorisation précise des poèmes.

La seconde citation est constituée du texte de l'épigramme que Lucien commande à Homère. Avant de quitter les morts et l'île des Bienheureux, le héros des Histoires Vraies dresse en effet une stèle commémorant son passage et son retour au monde des vivants, inversant la perspective des épitaphes dactyliques que l'on peut trouver, du temps de Lucien, dans les cimetières du Proche-Orient. Voici ce qu'elle dit:

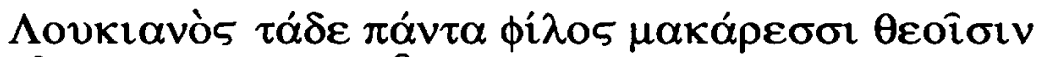

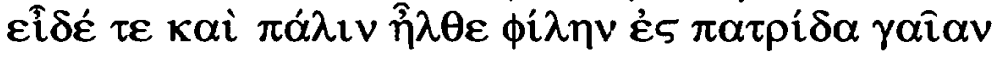

Lucien, ici, cher aux dieux bienheureux,

A tout vu et s'en est retourné dans sa patrie. (II, 28)

Les formules qui terminent les deux vers sont bien attestées dans la

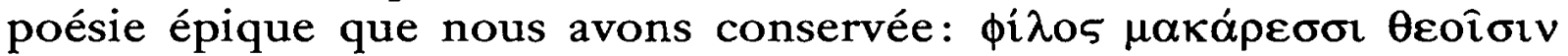
apparaît par exemple dans certains fragments, et en $O d$. I, 82 dans une

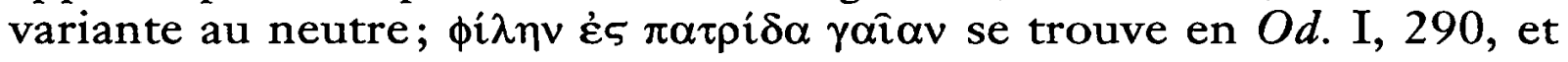

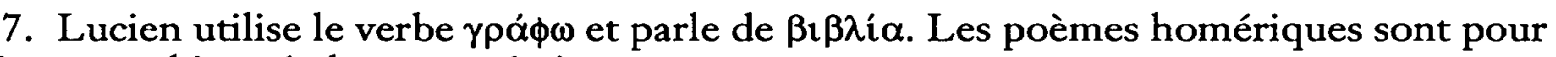
lui, sans ambiguiité, des textes écrits.

8. Voir Snell B., dir., Lexicon des frübgriechiscben Epos, Göttingen, 1979-, s.v.

9. Voir par ex. Od. IV , 268; XI, 629; XIV, 97, etc. 
constitue, après la coupe trochaïque, la variante de la formule très cou-

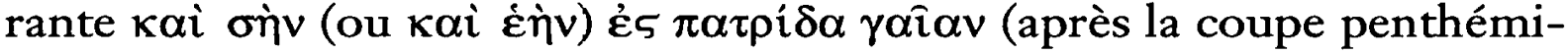
mère).

Nous n'avons pas trouvé d'équivalent du premier hémistiche du second vers. En revanche, le début du premier vers est très intéressant. Le nom de Lucien est évidemment non-homérique, mais il est très habilement intégré avant la formule $\tau \alpha ́ \delta \epsilon \epsilon \alpha ́ \nu \tau \alpha$, à une place où peut se trouver, dans la diction épique, un nom propre. Il suffit de comparer avec le vers 738 du chant $\Gamma$ de l'Odyssée :

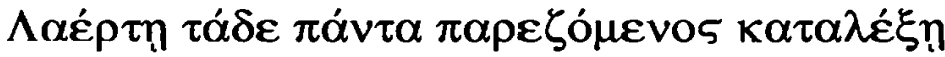

Lucien ne se contente donc pas de fabriquer un centon à partir de morceaux de vers épiques, mais se montre capable de créer des hémistiches inédits s'intégrant, par une permutation analogique, dans le système homérique. Son esprit, nourri d'épopée, non seulement mémorise les formules, mais semble aussi en saisir le mécanisme.

\section{Les scènes-types}

Si Lucien possède vraisemblablement du fonctionnement formulaire du vers épique une compréhension comparable à celle que nous avons acquise, quelle lecture fait-il des éléments traditionnels de la narration épique? ${ }^{10}$ Peut-on montrer que, dépassant le repérage des répétitions et des lieux communs homériques, qui servent de points d'accroche particulièrement efficaces à la parodie, il analyse comme nous le jeu de la variation sur le connu qui caractérise le fonctionnement des scènes typiques? ${ }^{11}$

Le récit des Histoires Vraies se prête aux répétitions, dans la mesure où la navigation fantaisiste de Lucien semble à première vue se dérouler sans autre logique que le plaisir de faire succéder un épisode à un autre ${ }^{12}$ : chaque île, chaque être marin rencontrés donnent lieu à un récit indépendant et fournissent l'occasion de descriptions ethnographiques, de scènes d'hospitalité ou de bataille. Certes, la durée de l'escale varie - de l'arrêt éclair dans l'Étoile du Matin aux longs séjours sur la Lune, dans le

10. Nous rejoignons, en ce qui concerne le maniement du vers formulaire, les conclusions d'O. Bouquiaux-Simon (op. cit., p. 359), qui a analysé les citations et allusions homériques dans l'ensemble de l'œuvre de Lucien. En revanche, sur le plan thématique, les scènes-types que nous allons examiner l'ont beaucoup moins intéressée que la reprise des épisodes majeurs comme la rencontre avec le Cyclope ou la descente aux Enfers, ce qui la conduit, nous semble-t-il, à sous-estimer la connaissance que Lucien possède de l'Odyssée (voir ibid. p. 354).

11. Voir J.M. Foley, op. cit. 
ventre de la baleine, ou sur l'île des Bienheureux -, mais au cours du voyage, les scènes de débarquement et d'embarquement, les scènes d'exploration se répètent. Or dans l'Odyssée, modèle de ces pérégrinations océaniques et célestes, ces scènes sont traditionnelles et formulaires. On peut donc légitimement se demander comment le texte des Histoires Vraies fait siennes ces scènes typiques, mais également si le processus mimétique concerne aussi les procédés mêmes de l'art traditionnel homérique, si l'écriture des Histoires Vraies cherche à les imiter en développant des stéréotypes propres et en jouant sur leur variation.

\section{Allusions aux scènes-types du voyage d'Ulysse}

Le vers par lequel Ulysse et ses compagnons reprennent la mer scande le récit chez les Phéaciens:

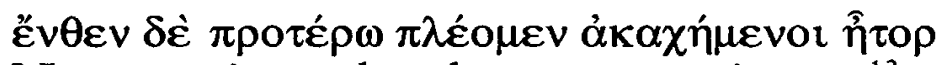

Nous reprîmes alors la mer avec tristesse ${ }^{13}$,

parfois accompagné de:

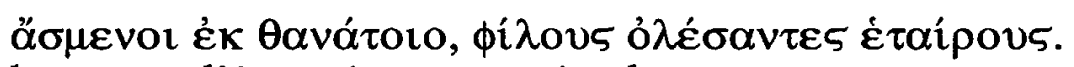

heureux d'être vivants, mais pleurant nos compagnons morts ${ }^{14}$

De même, Lucien rythme le récit de ses escales en répétant le verbe

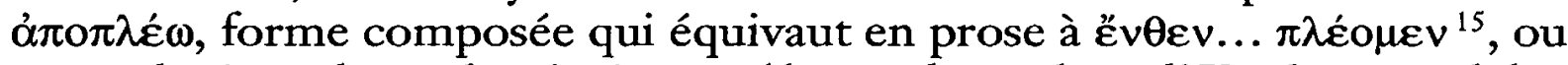

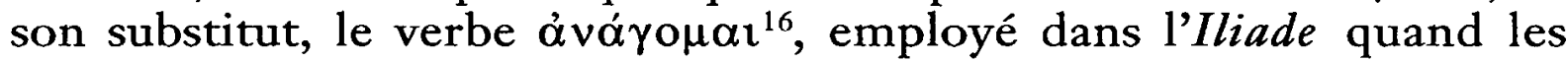
Achéens, Ulysse à leur tête, regagnent le large après avoir ramené Chryséis à son père (I, 478). L'absence de reprise du second hémistiche, qui exprime le sentiment de tristesse étreignant le cœur d'Ulysse, attire l'attention sur le caractère plaisant et fantaisiste du voyage de Lucien, qui contraste avec le pénible périple homérique imposé comme une épreuve au héros d'endurance.

Une fois que Lucien et ses marins ont embarqué, la durée de leur navigation s'exprime à la manière traditionnelle de l'épopée : «pendant $x$ jours,..., mais le jour suivant,... ${ }^{17} \gg$. Ce peut être l'occasion d'une allu-

12. La discussion sur la structure d'ensemble des Histoires Vraies déborde le cadre de notre étude. Voir G. Anderson, Studies in Lucian's Comic Fiction, Mnemosyne, suppl. 43, Leyde, 1976; B.P. Reardon, «Lucien et la fiction», in A. Billault, A. Buisson, éd., Lucien de Samosate: Actes du colloque de Lyon, Paris, 1994, p. 9-12.

13. IX, $62=\mathrm{IX}, 105=\mathrm{IX}, 565=\mathrm{X}, 77=\mathrm{X}, 133$. Les traductions de l'Odyssée sont empruntées à Philippe Jaccottet.

14. IX, 63, 566; X, 134 .

15. Cf. II, 2,$3 ; \mathrm{II}, 32$.

16. Cf. I, 6, 9; II, 29,35 .

17. Histoires Vraies, I, 6; I, 10. Cf. Od., V, 278-280, 388-392 ; IX, 82-84; X, 80-81 ; XII, 447-448. 
sion parodique manifeste. Sur le modèle du trajet d'Ulysse entre l'île de Calypso et la Phéacie, d'abord paisible puis effroyable:

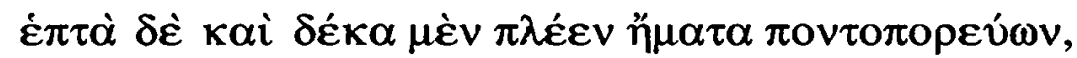

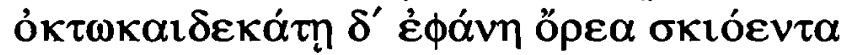

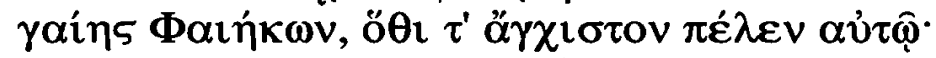

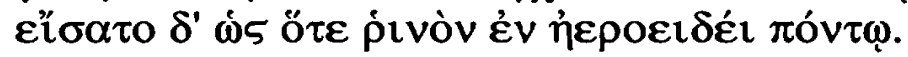

Dix-sept jours il cingla ainsi en haute mer; le dix-huitième jour apparurent les monts obscurs de Phéacie, du moins les plus proches de lui : dans la brumeuse mer, ils faisaient comme un bouclier (V, 278-280)

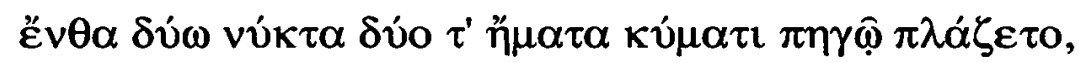

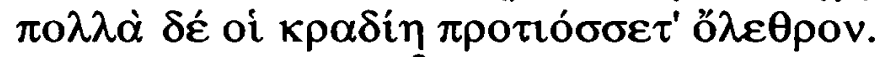

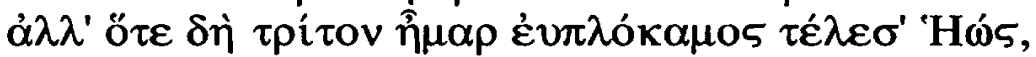

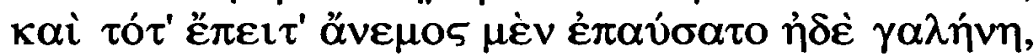

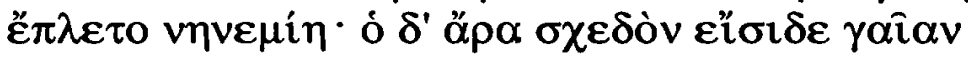

Alors, pendant deux jours et deux nuits, dans la houle, il dériva; son cour plus d'une fois crut voir la mort. Mais, quand l'aube bouclée amena le troisième jour, les vents enfin tombèrent; et dans le ciel, le calme se refit. Il vit la terre toute proche. (V, 288-392),

Lucien décrit le début agité de ses pérégrinations:

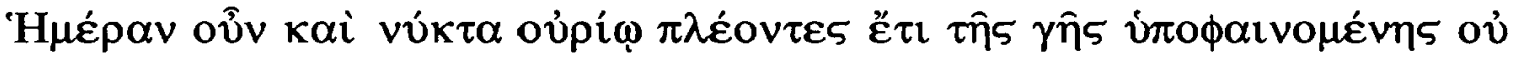

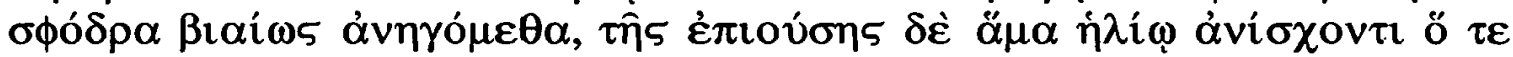

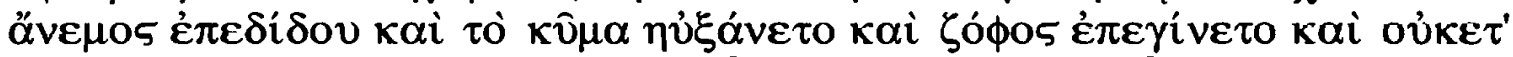

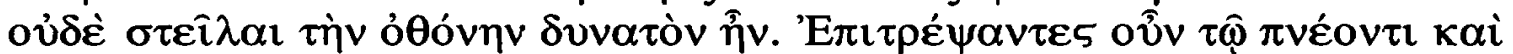

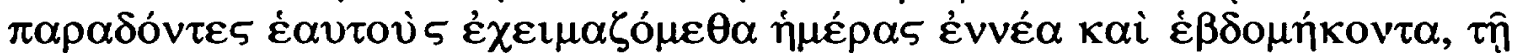

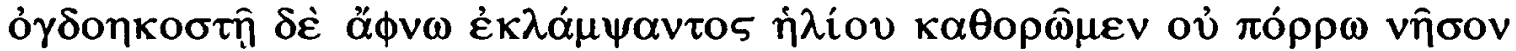

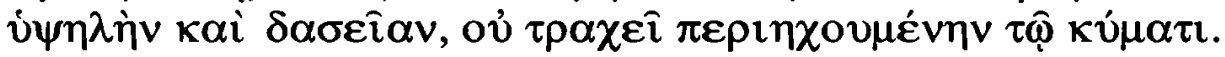

Naviguant pendant un jour et une nuit par vent favorable, encore en vue de la terre, nous gagnions le large fort calmement. Mais le lendemain, au lever du soleil, le vent augmenta, le flot s'enfla, la brume survint; il n'était même plus possible de carguer la voile. Nous nous confiâmes et nous abandonnâmes au vent qui soufflait. Nous fûmes ballottés par la tempête soixante-dix-neuf jours durant, mais soudain, le quatre-vingtième, le soleil brilla: nous apercevons, assez proche, une île élevée et boisée, entourée de vagues qui résonnaient doucement. $(I, 6)$

On retrouve dans le texte des Histoires Vraies tous les éléments de l'Odyssée : la structure narrative organisant la durée - les adjectifs numéraux permettant à Lucien une exagération comique -, la description de la dérive, la mention de l'aube, dépouillée des enjolivements de la formule 
concernant Éos, l'apparition d'une île proche, élevée, boisée, circulaire, mais sans la comparaison homérique ${ }^{18}$.

Le récit se poursuit avec le débarquement de Lucien et de ses compagnons, suivi d'un long repos:

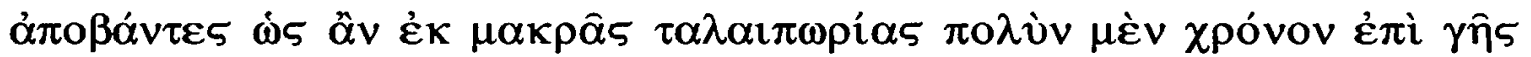

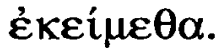

Nous débarquâmes, et, comme il est normal après de longues tribulations, nous restâmes étendus un long moment sur le sol. (I, 6)

Cette séquence est elle-même un souvenir de scènes formulaires de l'Odyssée :

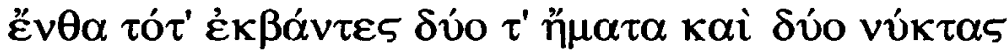

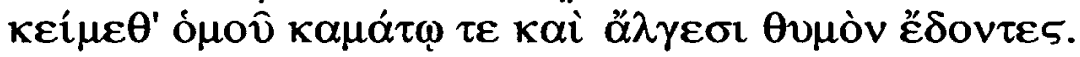

Là, deux jours et deux nuits, sans discontinuer,

nous restâmes couchés, rongés d'angoisse et de fatigue ${ }^{19}$.

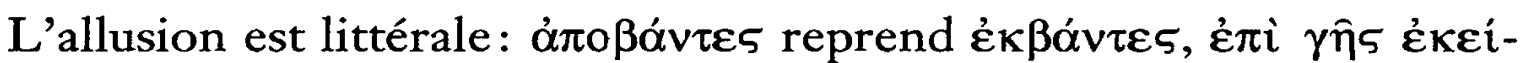

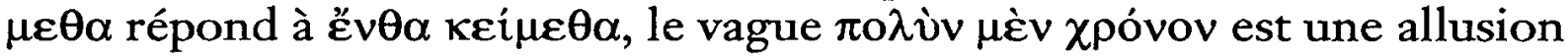

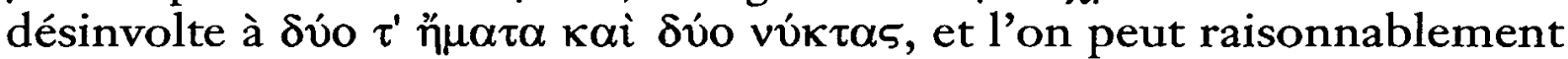

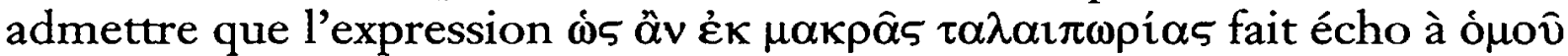

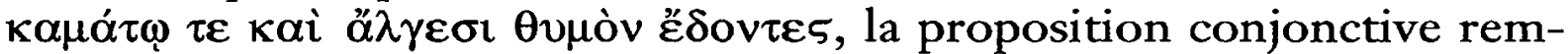
plaçant le participe circonstanciel. Mais par cette transformation, Lucien met l'accent sur la nécessité (ék est à la fois temporel et causal) qui lie la navigation éprouvante au besoin d'un long sommeil, alors que le texte homérique exprime davantage l'état d'esprit des héros. Ce faisant, l'imitateur justifie, à un second degré, l'apparition de la scène du sommeil après celle de la tempête; la nécessité n'est pas seulement factuelle, elle est aussi narrative. Il nous semble que nous avons là une sorte d'intervention ironique de Lucien, pointant la contrainte que lui impose le choix du modèle homérique et l'imitation du style formulaire. La séquence épique traditionnelle, qui associe le récit de tempête à celui du sommeil harassé, impose une sorte de causalité générale à laquelle le parodiste ne saurait se soustraire ${ }^{20}$.

18. Pour le modèle de la tempête, voir en particulier XII, 402 et $s q$.

19. IX, 74-75 = X, 142-143.

20. D. van Mal-Maeder examine à travers cet exemple la manière dont Lucien «prosifie» la poésie d'Homère et utilise ses «tics narratifs» pour donner à son propre récit le «caractère vivant» et la «patine de vraisemblable» que ces scènes stéréotypées conféraient, selon elle, à l'Odyssée (p. 129-130), mais elle n'envisage pas la formularité homérique - et encore moins sa reprise par Lucien - comme un procédé dynamique de création. Voir «Les détournements homériques dans l'Histoire Vraie de Lucien: Le rapatriement d'une tradition littéraire», Etudes de lettres, 1992-2, Lausanne, p. 123-146. 
Un autre vers homérique est employé pour dire le débarquement:

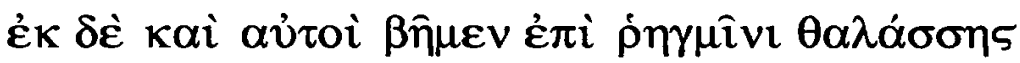

Nous mîmes pied à terre sur la frange des brisants ${ }^{21}$.

Il est précédé dans l'Odyssée de la mention du bateau qu'on échoue ou des voiles que l'on cargue, et devient chez Lucien, quand il aborde sur la Lune ou l'île des Bienheureux:

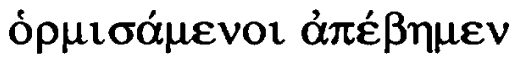

Après nous être amarrés, nous débarquâmes $(I, 10)$ et

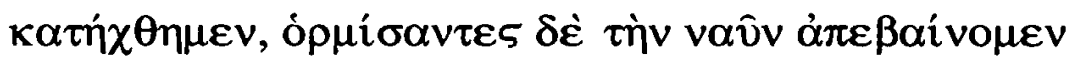

Nous abordâmes, et, après mis le vaisseau à l'ancre, nous débarquâmes. (II, 6)

Une fois à terre, Ulysse comme Lucien se livrent à des opérations indispensables: faire de l'eau, se ravitailler, manger, dormir sur la grève.

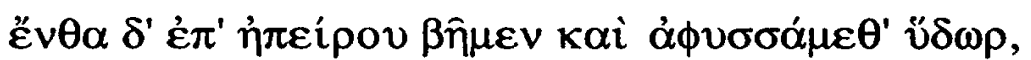

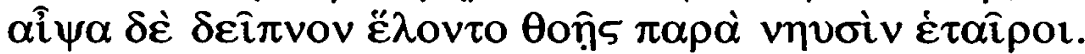

On descendit à terre, on refit provision d'eau fraîche, on mangea vite auprès des rapides navires ${ }^{22}$.

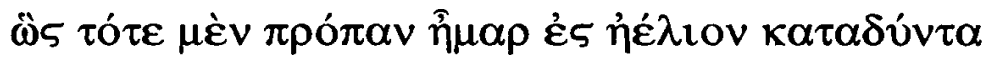

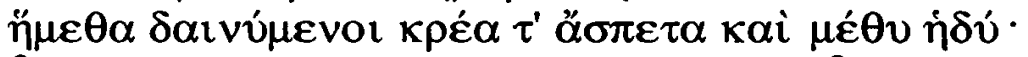

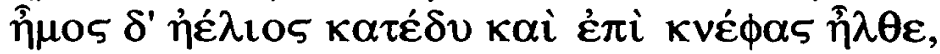

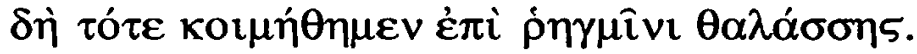

Alors, de tout le jour et jusqu'au coucher du soleil, nous restâmes assis devant force viande et vin doux.

Le soleil se coucha, le crépuscule vint, et nous nous étendîmes sur la frange des brisants ${ }^{23}$.

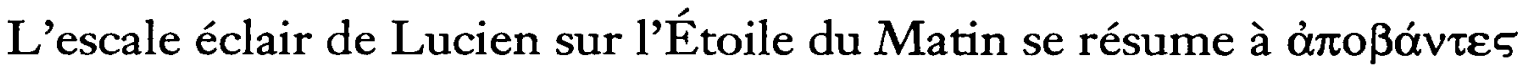

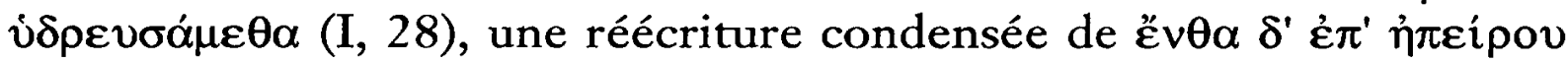

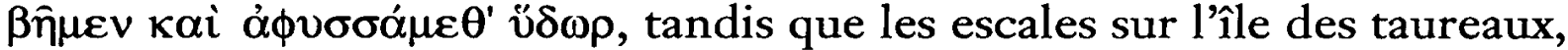
puis des Bucéphales, associent la recherche de l'eau et de la nourriture :

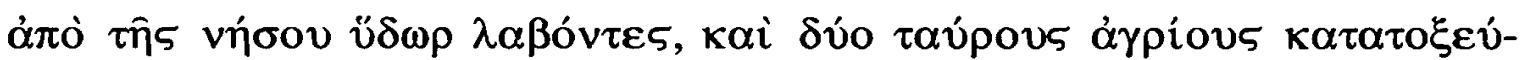

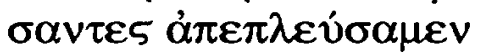

21. IX, $150=\mathrm{IX}, 547$.

22. Od. IX, $85-86=\mathrm{X}, 56-57=\mathrm{X}, 100-101$

23. IX, 556-559 = X, 183-186 = XII, 29-32 (avec une variation sur le dernier vers) = IX, 161-162 + 168-169. Voir aussi X, 6-7 pour les deux derniers vers. 


\section{Histoires vraies de Lucien}

Après avoir fait provision d'eau sur l'île, nous abattîmes avec nos flèches deux taureaux sauvages, et nous reprîmes la mer. (II, 3)

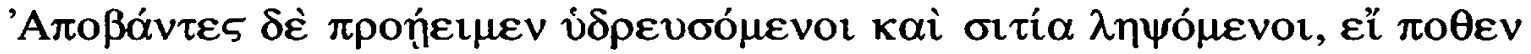

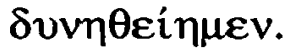

Nous débarquâmes et nous nous avançâmes pour faire de l'eau et rapporter des provisions, si toutefois c'était possible. (II, 44)

À la fin de l'épisode de l'île des Songes, que Lucien quitte dans la pré-

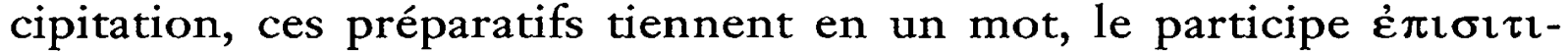
$\sigma \alpha ́ \mu \varepsilon v o r$, «après nous être approvisionnés» (II, 35).

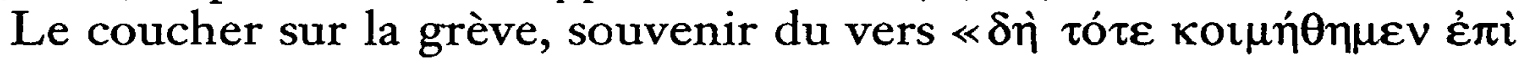
$\dot{\rho} \eta \gamma \mu \hat{\imath} v \imath \theta \alpha \lambda \alpha \dot{\sigma} \sigma \sigma \eta 5 »$, est également un thème récurrent, susceptible d'être associé au précédent:

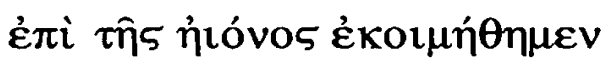

Nous nous couchâmes pour dormir sur la grève. (II, 36)

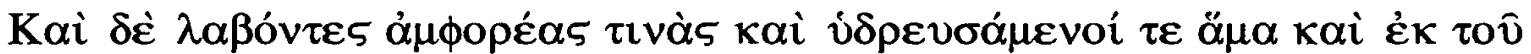

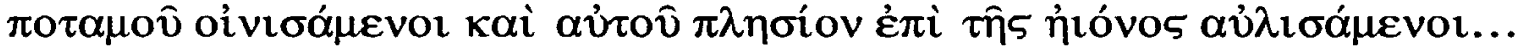

Puis, ayant avec des amphores fait provision d'eau et en même temps tiré du vin du fleuve, nous campâmes près de celui-ci sur la grève. (I, 9)

Ce dernier exemple est particulièrement intéressant dans la manière dont il se démarque du modèle odysséen pour mieux affirmer son originalité : il s'agit de l'escale sur l'île des femmes-vignes, où coule un remar-

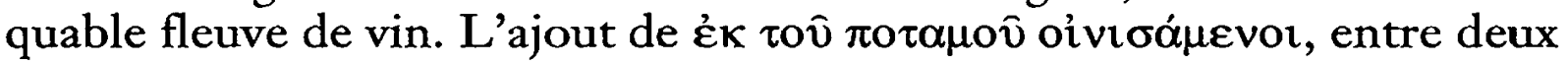
allusions très claires aux vers homériques, signale l'invention du récit de Lucien tout en l'intégrant à la scène-type comme pourrait le faire un aède.

Dernier élément récurrent des séquences d'abordage, le motif de l'exploration. Comme Ulysse, Lucien est projeté dans un monde inconnu, et les nécessités du ravitaillement, ajoutées à la curiosité, le poussent à pénétrer au cœur des îles où le hasard le mène. Dans l'Odyssée, il existe deux scènes-types qui peuvent se combiner, comme dans l'épisode des Lestrygons. Dans l'une, Ulysse choisit les hommes qui partiront en reconnaissance, avec ou sans lui :

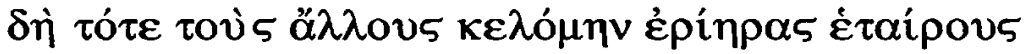

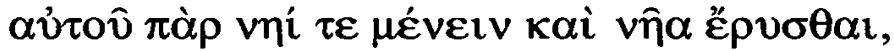
$\alpha$ $\beta \hat{\eta} \mathrm{v}$.

Alors, je commandai à mes fidèles compagnons de rester auprès du vaisseau pour le garder.

Puis, lorsque j'eus choisi les douze plus braves d'entre eux, je partis. (IX, 193-196) 


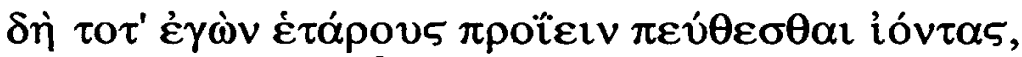

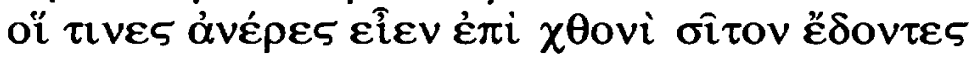

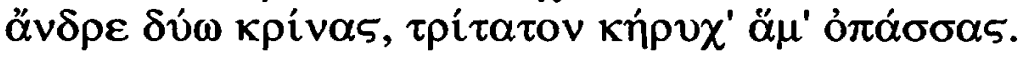

J'envoyai de mes gens pour s'informer quels étaient les mangeurs de pain qui vivaient là ; j'en choisis deux, auxquels j'adjoignis un héraut ${ }^{24}$.

Mais il arrive aussi qu'Ulysse ait au préalable repéré les lieux:

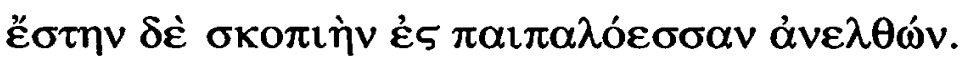

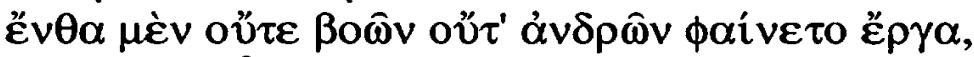

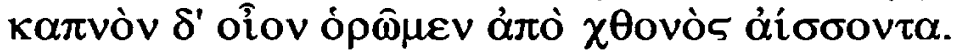

Je montai sur une roche pour guetter.

De là n'apparaissaient ni labourages ni jardins, on voyait seulement des fumées s'élever de terre. (X, 97-99)

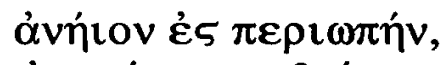

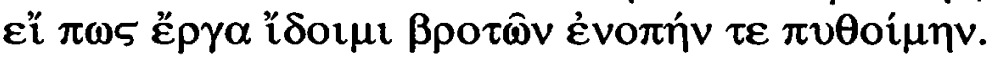

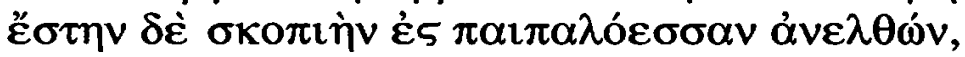

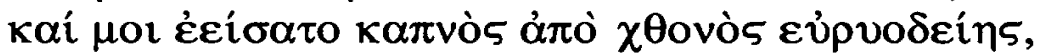

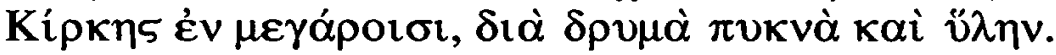

Je grimpai sur une hauteur dans l'espoir de voir des jardins, d'ouïr des voix. Je montai donc sur une roche pour guetter et vis une fumée monter du sol aux grandes voies, du palais de Circé dans les bois et les chênes denses. (X, 146-150)

Le premier mouvement d'Ulysse apercevant de la fumée chez Circé est de redescendre au bateau pour envoyer des hommes à la rencontre des habitants de l'île ${ }^{25}$; nul doute que nous aurions trouvé alors les vers formulaires cités plus haut. Mais il est interrompu par l'irruption d'un cerf, mis sur son chemin par «quelque divinité» pour lui servir de proie - et détourner son esprit de son but. En effet, après le festin que lui permet cette chasse, il laisse passer la nuit. Au matin, il décide, instruit par les mésaventures précédentes, de diviser ses hommes en deux contingents, l'un partant chez Circé, l'autre restant aux navires, ce qui s'avèrera salutaire et ménage une scène dramatique à souhait, celle du tirage au sort pour savoir quel groupe prendra le risque de s'enfoncer dans l'île ${ }^{26}$.

On retrouve chez Lucien, comme on s'y attend, des allusions à ces passages. Lors du débarquement sur la Lune, les scènes homériques sont

24. IX, 88-90 (Lotophages) $=\mathrm{X}, 100-102$ (Lestrygons). Pour une variante abrégée, voir X, 59.

25. $\mathrm{X}, 151-155$.

26. $X, 187-209$. 
amalgamées en une courte phrase, et néanmoins clairement reconnaissables :

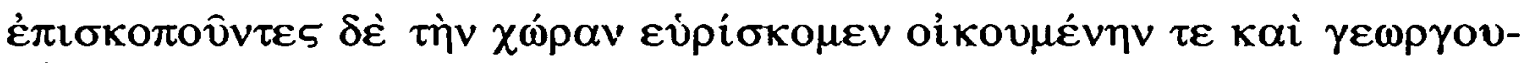

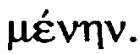

En examinant le pays, nous découvrons qu'il était habité et cultivé. (I, 10)

Du point de vue textuel, la référence est la scène de reconnaissance d'Ulysse seul, bien que l'action soit collective. Le participe

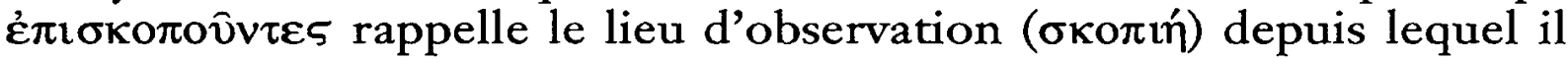
prend ses repères, et le but de l'observation, voir si le pays est habité et

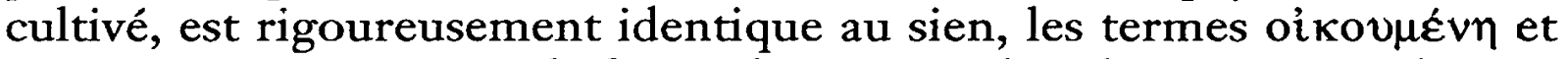
$\gamma \varepsilon \omega \rho \gamma o v \mu \varepsilon ́ v \eta$ exprimant de façon abstraite ce dont le personnage homé-

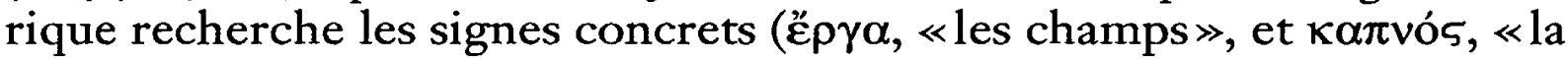
fumée $\gg)$.

Dans un contexte un peu différent, Lucien, arrêté dans sa navigation par ce qui semble être une forêt flottante, tente d'avoir une meilleure vue du phénomène:

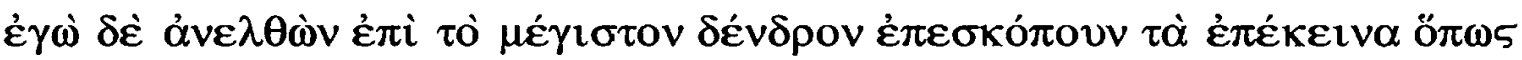

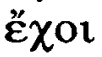

Je montai donc sur le plus haut des arbres pour examiner ce qu'il y avait au-delà ${ }^{27}$.

La légère modification de l'original homérique est une adaptation rendue nécessaire par le paysage dans lequel se trouve Lucien: point de roche élevée dans cette étrange contrée entièrement végétale, mais la cime des arbres offre un poste d'observation tout à fait analogue, tout en permettant de souligner la spécificité de l'épisode.

Sur l'île des femmes-vignes, comme dans le ventre de la baleine, le modèle est celui du choix des explorateurs :

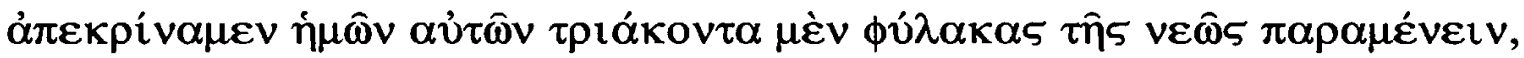

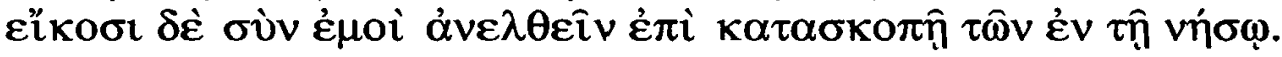

Nous choisîmes trente d'entre nous pour rester à la garde du bateau, et vingt pour m'accompagner vers l'intérieur afin d'observer les particularités de l'île. $(I, 6)$

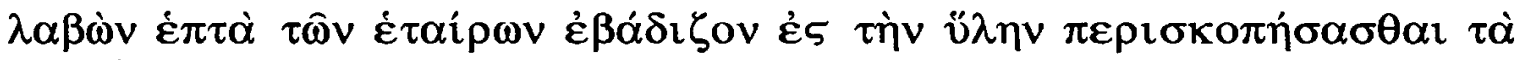
$\pi \alpha v \tau \alpha \grave{~ B o v \lambda o ́ \mu \varepsilon v o s ~}$

Je pris avec moi sept compagnons pour marcher vers la forêt, car je voulais inspecter tous les environs. (I, 32)

27. II, 42. 
Au participe кpívas, que l'on trouve dans l'Odyssée, correspondent la forme composée $\dot{\alpha} \pi \epsilon \kappa \rho^{\prime} \nu \alpha \mu \epsilon \nu$ et le participe $\lambda \alpha \beta \tilde{\omega} v$, à $\beta \hat{\eta} v$ la forme $\dot{\varepsilon} \beta \alpha^{\prime}-$ $\delta i \zeta o v$, et la mention des nombres est à nouveau utilisée comme prétexte à l'hyperbole parodique. Lucien s'amuse également avec les signes de vie humaine qui préoccupent Ulysse: son héros, au cours de la reconnaissance à l'intérieur de la baleine, entend un chien aboyer (voilà pour les voix qu'Ulysse espère entendre du haut de son observatoire), aperçoit de la fumée, et tombe nez à nez avec un vieillard et son fils en train de...

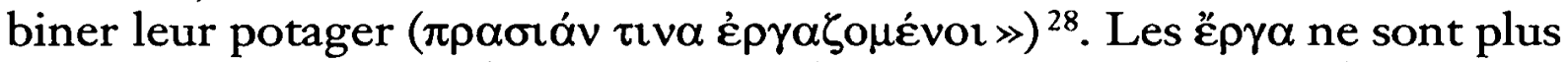
seulement un indice de la présence d'êtres civilisés, mais le décor dans lequel les êtres en question apparaissent à Lucien.

Celui-ci introduit néanmoins, dans les deux épisodes, des éléments nouveaux. L'exploration le conduit en effet avec ses compagnons à s'éloigner de la grève et à s'enfoncer au cœur d'une forêt, où ils découvrent, dans le cas du pays des femmes-vignes, une stèle mentionnant Dionysos et Héraclès, et dans le cas de la baleine, un temple de Poséidon et une série de stèles funéraires. Ces objets, bien qu'énigmatiques, signalent eux aussi une présence humaine et, bien entendu, hellénophone. Lucien introduit dans le schéma homérique des données anachroniques, mais il s'agit moins d'une innovation que d'une actualisation, car on comprend aisément que pour lui, l'écriture appartienne désormais aux traits caractéristiques de la civilisation, au même titre que l'usage du feu et l'agriculture.

Ces quelques exemples nous persuadent un peu plus, si besoin était, que Lucien maîtrise la matière de l'Odyssée jusque dans ses détails, mais ils démontrent surtout qu'il est extrêmement sensible à l'effet structurant des scènes-types du voyage d'Ulysse et qu'il cherche, en adaptant ces séquences, à rythmer son propre récit comme le faisait Homère, sans craindre la répétition. Dépouillé de tout ce qui fait son caractère poétique spécifique (épithètes, comparaisons, formes verbales simples et sans augment, etc.), le modèle épique, dans ses récurrences, fournit des articulations au récit des Histoires Vraies et lui confere à son tour, par le biais de l'imitation, une certaine formularité. Mais Lucien ne s'arrête pas à la transposition des scènes-types homériques: il imite le processus même de création des aèdes et invente des variations à partir du cadre narratif que l'imitation des scènes-types lui a imposé.

28. I, 32-33. 


\section{L'art formulaire de Lucien}

Lucien, nous l'avons vu, n'hésite pas à augmenter la scène-type homérique d'éléments de son cru. Prenons par exemple l'approvisionnement en vin sur l'île des femmes-vignes: il s'intercale entre deux motifs formulaires, l'approvisionnement en eau et le repas sur la grève avant le départ. Variation par rapport au modèle homérique, cette innovation sert également à singulariser l'épisode à l'intérieur de la narration des Histoires Vraies, dans la mesure où puiser de l'eau avant de s'embarquer est une scène récurrente du voyage de Lucien. La structure de l'Odyssée servant de modèle à celle des IIisLoires Vruies, tout écart par rapport à la première, s'il n'est pas généralisé (comme l'est par exemple l'intégration des signes écrits comme indice de la vie humaine), devient potentiellement variation significative par rapport à la seconde. On peut donc dire que Lucien utilise la formularité de la scène où l'on fait de l'eau pour mettre en relief la scène isolée où l'on «fait du vin» en puisant au fleuve prodigieux.

Les épisodes de l'île des taureaux et de l'île des Bucéphales donnent lieu à un autre type de jeu formulaire. Tous deux font allusion à la scènetype «ravitaillement en eau et en vivres + départ ${ }^{29} \gg$. Dans le premier, l'escale est très courte, et se résume à faire de l'eau et à tuer deux taureaux avant de rembarquer ${ }^{30}$. Dans le second, la narration est nettement plus développée, mais commence par l'intention de trouver de l'eau et des vivres «si toutefois c'était possible ${ }^{31} \gg$. Cette proposition hypothétique ouvre un espace à la variation du récit, et titille le lecteur en lui suggérant que cette fois-ci, les choses seront plus compliquées que d'ordinaire. Lucien et ses compagnons, de fait, trouvent immédiatement de l'eau, mais n'aperçoivent aucune proie potentielle. Des mugissements, toutefois, leur parviennent, et, croyant tomber sur un troupeau de bœufs (auquel cas l'épisode se serait ramené à celui de l'île des taureaux), ils s'enfoncent dans l'île. Mal leur en prend: elle est habitée par des hommes à tête cornue, hostiles et anthropophages, qui leur donnent la chasse. Réfugiés près de leurs bateaux, ils s'arment et repartent pour une expédition punitive. Leur victoire est totale, et ils massacrent tous les Bucéphales qui les avaient attaqués sauf deux, qu'ils font prisonniers. Lucien doit empêcher ses compagnons de les tuer - manifestement pour les manger ${ }^{32}$, , ce qui aurait également créé un parallèle avec les deux

29. Voir ci-dessus, p. 226.

30. II, 3 .

31. II, 44.

32. La phrase précédente dit: «Mais nous n'avions pas trouvé de vivres», et est articulée à la tentative de meurtre par un oûv. Il y a dans ce lien entre le manque de vivres et la 
taureaux sauvages tués lors de l'autre escale, à ceci près que les Bucé-

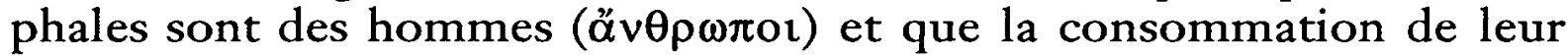
chair aurait mis Lucien et ses hommes dans une situation de sacrilège que les Histoires Vraies évitent soigneusement - trop sérieux, sans doute ! Le récit se termine par l'échange de ces otages contre une nourriture abondante et moralement tout à fait acceptable, même si elle comprend la viande d'étonnantes biches à trois pattes. De cette manière, la séquence-type, un temps contrariée par la narration de la rencontre avec les Bucéphales et du combat qui s'ensuit, se résout harmonieusement: on peut repartir, les cales pleines de provisions.

Dans la configuration du livre II, l'épisode de l'île des taureaux, nous semble-t-il, prépare le lecteur à la singularité de celui des Bucéphales. Il fournit en quelque sorte le thème (l'eau, les deux taureaux, le ravitaillement) sur lequel Lucien va ensuite composer une variation développée, recherchant des effets d'attente et de surprise dans les écarts entre les deux récits.

Les scènes d'exploration, enfin, offrent l'exemple d'un stéréotype créé par Lucien - sur la base d'une scène-type homérique - et utilisé par lui de manière dynamique et féconde. Les éléments récurrents de la découverte des îles sont, nous l'avons dit, le départ en groupe vers l'intérieur du pays, généralement boisé, à la recherche d'eau et de nourriture, et l'observation de signes mystérieux témoignant de la présence de vie humaine. Il en va ainsi sur l'île des femmes-vignes, celle des Bucéphales, ou dans le ventre de la baleine. La visite à Calypso, elle, réserve une surprise, ou plutôt une déception. Lucien aborde Ogygie, et commence par lire, avec beaucoup d'indiscrétion, la lettre qu'Ulysse l'a prié de remettre à la nymphe. Passé ce délectable pastiche, la narration semble s'orienter vers un récit d'exploration et nous montre Lucien s'enfonçant dans l'île. Mais cela tourne court, car nous ne sommes pas en terrain inconnu. Le héros trouve sans peine la grotte de Calypso, qu'il se refuse à décrire: elle est, dit-il avec une certaine paresse, « exactement telle qu'Homère l'a dépeinte ${ }^{33} \gg$. La rupture du jeu formulaire ménage ici la place à l'établissement d'un rapport de connivence entre l'auteur et son lecteur, chargé de pallier, par sa culture, la réticence ou la désinvolture de l'imitateur. Dans le même temps, l'absence de la scène attendue - la découverte progressive d'une île énigmatique - rend plus sensible la formularité que Lucien a instaurée ailleurs.

tentation de la nourriture interdite une réminiscence évidente de l'épisode odysséen des boufs du Soleil.

33. II, 36. La confirmation de la description homérique a plus de sel encore si on la met en rapport avec le tableau de la cité des Songes, introduit par: «Je veux vous parler de cette ville, car aucun autre écrivain n'en a traité, et Homère, qui est bien le seul à la mentionner, n'est pas très exact dans son récit.» (II, 32). 
La variation ludique sur cette séquence atteint un sommet avec la découverte de l'île des impies. Présentée d'emblée comme l'inverse de l'île des Bienheureux (une odeur nauséabonde s'en dégage, qui contraste vivement avec le délicieux parfum du séjour des héros) ${ }^{34}$, elle donne à Lucien l'occasion de renverser le stéréotype qu'il a mis en place tout au long des Histoires Vraies. Le récit commence sous forme négative: «nous n'abordâmes pas [les îles des impies] », sauf une. Celle-ci, contrairement à l'île des Bienheureux qui offre des rivages et des ports accueillants, présente des côtes abruptes, déchiquetées. Elle est par ailleurs sans arbre et

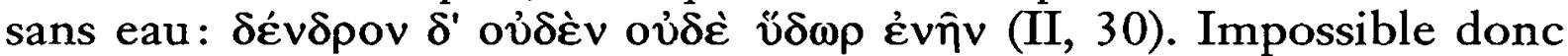
pour le narrateur de développer la scène habituelle. Il ne peut être question ni de ravitaillement, ni d'exploration des bois. Comme chez les femmes-vignes, le pays se distingue par la nature prodigieuse de ses fleuves, mais ceux-ci sont de boue, de sang et de feu, et Lucien ne saurait y puiser comme il l'a fait au fleuve de vin; il ne peut donc faire entrer ces détails dans le cadre du récit d'approvisionnement. Sous la conduite d'un guide, les voyageurs poursuivent leur visite, observent le sort des rois et des quidams, et se font expliquer les raisons de leur présence. Alors que le tableau est assez général et procède par catégories sans s'arrêter aux châtiments subis, un seul des damnés reçoit un traitement détaillé :

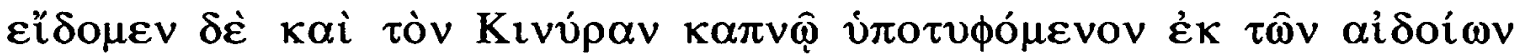

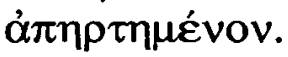

Nous vîmes aussi Kinyras enfumé par le feu au-dessus duquel il était suspendu par les parties. (II, 31)

Kinyras est le fils de Skintharos, le vieillard rencontré dans le ventre de la baleine; après avoir quitté l'estomac du monstre, il avait rejoint les rangs des compagnons de Lucien et l'avait accompagné sur l'île des Bienheureux. S'il jouit chez les impies d'un traitement de faveur, c'est pour avoir succombé aux charmes de l'incorrigible Hélène et l'avoir enlevée. Le tableau qu'il offre aux visiteurs frappe d'emblée, si on se place dans la perspective que nous avons établie. Là où la formularité des scènes d'exploration nous fait attendre la découverte d'une fumée signalant une présence humaine, nous avons bien de la fumée et un homme celui-là même que l'on découvrait binant son potager dans l'estomac du cétacé. Mais, suspendu comme une pièce de viande, il a perdu toute dignité humaine, et le feu infernal n'a plus rien du rassurant foyer domestique. Lucien, au moment où il s'affranchit du cadre formulaire pour décrire un lieu de mort où tout s'inverse, garde manifestement ce

34. Pour les descriptions opposées des deux îles, voir II, 5 (Bienheureux) et II, 30-31 (impies). 
cadre à l'esprit et s'amuse du bouleversement qu'il lui fait subir. Le plus extraordinaire est peut-être que, ce faisant, il se montre plus consciencieux imitateur d'Homère que jamais. En effet, les scènes-types du voyage d'Ulysse connaissent elles aussi une parenthèse : l'abordage au pays des morts est raconté sans recours aux vers formulaires que nous avons examinés ${ }^{35}$. Le ravitaillement et le repas pris sur la grève par Ulysse et ses compagnons sont remplacés par la mention des bêtes que l'on débarque pour offrir un repas sacrificiel aux habitants du lieu, les fantômes des morts. La non-formularité souligne ainsi le caractère exceptionnel et anormal du monde avec lequel Ulysse entre en contact. La formule reprend ses droits lorsque le héros rembarque et ordonne à ses hommes de s'asseoir à la rame pour rejoindre l'univers des vivants ${ }^{36}$.

Au terme de cette étude des scènes récurrentes du voyage imaginaire de Lucien, il nous semble avoir montré que l'imitation de l'Odyssée dans les Histoires Vraies va bien au-delà d'une simple reprise ludique et parodique de thèmes familiers. La connaissance intime que Lucien possède de l'épopée se traduit, outre par la précision et la finesse de ses allusions, par la capacité à se couler dans le système formulaire et à le reprendre à son compte pour créer quelque chose d'inédit. Les répétitions auxquelles le récit s'abandonne, et qui ont gêné les critiques comme les redites homériques ont pu le faire, sont en fait le signe que la narration épouse la structure formulaire odysséenne et adapte ses scènes-types. Si, dans sa pratique de la mimésis, Lucien néglige de tirer parti de certains traits stylistiques des poèmes d'Homère comme les épithètes composées ou les comparaisons, il met à nu les règles qui gouvernent le fonctionnement de la narration épique et les définit ainsi comme leur caractéristique essentielle.

35. $\mathrm{XI}, 14 s q$.

36. XI, 636-638. 